\title{
Tecnologías convergentes para la enseñanza: Realidad Aumentada, BYOD, Flipped Classroom
}

\section{Converging technologies for teaching: Augmented Reality, BYOD, Flipped Classroom}

\author{
José Manuel Sánchez-García \\ Universidad de Sevilla. Sevilla, España \\ josesanchez@us.es \\ Purificación Toledo-Morales \\ Universidad de Sevilla. Sevilla, España \\ ptoledo@us.es
}

\begin{abstract}
Resumen
Conocer qué tecnologías surgirán en los próximos años interesa para de esta forma abordar los problemas que surgirán en las aulas educativas. La confluencia de tendencias como el Bring Your Own Devide (BYOD), Realidad Aumentada (RA) y Flipped Classroom (FC), darán lugar a cambios que es interesante investigar, saber de antemano si se puede producir esta convergencia y qué valor puede tener en el proceso de enseñanza y aprendizaje. Para ello es conveniente abordar los retos que supone esta integración, qué legislación regula el acceso de dispositivos electrónicos en clase, ya sea para su utilización en educación superior, como en bachillerato, educación secundaria, primaria e infantil. Interesa conocer cuáles son los retos que plantea el acceso a estos dispositivos en los centros por parte de profesorado y alumnado. Así como qué materiales son para ello más adecuados en aspectos referidos a dispositivos de hardware y la elaboración de contenidos por parte de profesores y empresas del ámbito de la educación. En aspectos relativos al uso de dispositivos y contenidos, la mejora de la calidad educativa en los diferentes niveles en los que se incorporen contenidos de este tipo y las ventajas, desventajas y riesgos que supone su utilización en los mismos.
\end{abstract}

Palabras clave

Bring Your Own Devide (BYOD), Realidad Aumentada (RA), Entornos de aprendizaje, Aplicaciones Educativas, Implantación.

\begin{abstract}
Knowing that technology will emerge in the next years we are interested to deal with problems that arise in this way in our classrooms. The confluence of trends such as the Bring Your Own Devide (BYOD) and Augmented Reality (AR), will give place to change that is interesting to know and investigate, knowing in advance if this convergence may occur, and that value can have in our classrooms is the end of this paper. Thus is convenient to approach the challenges posed by this integration that legislation regulates access of electronic device to classrooms, either for your use in universities, as in high school, that and education formative stages, child education and primary. Which are the challenges in how much access to these devices by centers, teachers and students. Those materials are so more appropriate aspects referring to hardware devices such as in terms of the management and processing of contents by teachers and companies of the field of education. In the relative use of devices and content aspects, the improvement of the quality of education in the different educational levels in implanted them content of this type, and about the advantages, disadvantages and risks involved in their use in them.
\end{abstract}

Key words

Bring Your Own Devide (BYOD), Augmented Reality (AR), Learning Environments, Educational Applications, Implantation. 


\section{Introducción}

En el ámbito educativo no son raros los casos en los que convergen diferentes tecnologías. En el uso de los medios se ha pasado del vetusto proyector de diapositivas, de transparencias o de vídeo, a las pizarras digitales interactivas... elementos que aúnan el uso de imagen, audio, programas, etc. Cuando se toman diferentes tecnologías para integrarlas y darles un uso didáctico, exceptuando los contenidos, rara vez surgen en primer lugar dentro del propio ámbito educativo. Ha sufrido esta convergencia el periodismo que ha tenido que adaptarse a los nuevos formatos que presentan las telecomunicaciones y que suponen un cambio en las redacciones, la formación de los periodistas, nuevas destrezas en redacción, locución, edición, modelo de negocio, etc... para producir en distintos soportes y plataformas (Peña, Lazkano \& García, 2016).

Se considera que muchas de las herramientas actuales que encontramos en el aula son producto de la implantación de tecnologías que se han vuelto cotidianas, están al alcance de todos y son aceptadas por toda la sociedad. En su día se guardaron como secretos el paso del pergamino al papel, la aparición y uso del libro, la invención de la imprenta, el lápiz, el bolígrafo... por enfocarlo desde los elementos más cotidianos. Hoy la integración proviene de la implantación en las aulas de ordenadores, pizarras digitales interactivas y Tablets o Smartphone. La llegada de estos dispositivos parece abocarnos a cambios tanto en el rol docente como en las nuevas formas de aprender de nuestros alumnos, tendencias como el uso de Bring Your Own Devide (BYOD), la Realidad Aumentada (RA) y las Flipped Classroom (FC), y que impulsan la utilización en el marco educativo de dispositivos móviles y acceso a las redes. Se han de definir nuevas formas de abordar el aprendizaje, el diseño curricular, el proceso de enseñanzaaprendizaje y el uso de las infraestructuras disponibles (Cochrane, Antonczak, Keegan \& Narayan, 2014). Procesos en los que media la tecnología, que convergen en la escuela y crean sinergias que van más allá de las meras dotaciones. Es cotidiana la frase ¡Sacad papel y lápiz! pero estos elementos no convergieron en un aula hasta el último cuarto del siglo XIX, en la actualidad estos elementos presentan usos cotidianos. Esto sucederá en los próximos años con algunas de las tecnologías que están surgiendo hoy, las que ahora podemos considerar emergentes.

\section{Realidad Aumentada, BYOD y cambios en el rol docente con el Flipped Classroom. ¿Qué son y cómo los usaremos?}

Para entender mejor la convergencia de algunos de los elementos es necesario conocer y aclarar los términos con los que se trabaja, conocer definiciones concretas y prácticas sobre cuál es su uso en ámbitos educativos.

\subsection{En la encrucijada de la realidad aumentada}

Para Johnson, Adams Becker, Estrada y Freeman (2015), la adecuación de la RA a la docencia ya está teniendo lugar, y puede ser útil para reforzar los departamentos de diferentes materias, como por ejemplo la ingeniería, sistemas de comunicación entre diferentes especialidades o favoreciendo la creación grupos multidisciplinares que determinen la mejor forma de presentar los contenidos de cada materia. Encontramos 
ejemplos de este cambio en diferentes instituciones: PBS EducationShift, en la Universidad de West Virginia que plantea la creación del Media and Innovation Center o el Houston Community College en el West Houston Institute, que están habilitando espacios para la producción de medios de RA, laboratorio de narrativa digital y de colaboración y estudio de la RA. Que tienen en común ser lugares para la colaboración multidisciplinar que puede contribuir a un aprendizaje significativo (Johnson, Adams Becker, Estrada \& Freeman, 2015).

La Realidad Aumentada superpone a la realidad imágenes virtuales, estas pueden ser modelos 3D o información sobre objetos, sitios, iconos, personas o llugares y se genera mediante medios informáticos. Para Durlach y Mavor (1995) es la combinación entre entornos reales y elementos de Realidad Virtual (RV), definición muy amplia y que integra las características de esta última. De Pedro (2011) aprecia que la RA completa la percepción del mundo real aportando al usuario información creando una realidad mixta. No sustituye el mundo real por una versión virtual, completa con información lo que vemos mediante la intermediación de un dispositivo informático con una cámara integrada. Barfield (2015) arguyen que el usuario ve el mundo real e interactúa con la información que le facilitan los dispositivos, para Prendes (2015: 187) "la realidad aumentada es una prometedora tecnología, ya presente en muchas aulas, que puede ayudar a mejorar el proceso de enseñanza-aprendizaje". De Pedro (2011) considera que nos permite interactuar con el mundo real dándonos información, combinando la realidad física con elementos virtuales en tiempo real. Mientras que Basogain, Olabe, Espinosa, Rouèche y Olabe (2007: 1) la definen en términos en los que "la realidad aumentada no reemplaza el mundo real por uno virtual, sino al contrario, mantiene el mundo real que ve el usuario complementándolo con información virtual superpuesta al real. El usuario nunca pierde el contacto con el mundo real que tiene al alcance de su vista y al mismo tiempo puede interactuar con la información virtual superpuesta", definiciones similares en muchos aspectos.

Azuma (1997) en fechas tempranas para esta tecnología identificó tres características principales: a) Combina lo real y lo virtual, b) Interactiva y en tiempo real, y c) Registrada en 3D. En cuanto a la creación de contenidos, los programas que permiten generar información para entorno de RA pueden integrar información en $2 \mathrm{~d}$, video o texto como fuente de información, o páginas web con información textual y gráfica. Para Prendes (2015) y Johnson, Adams Becker, Estrada y Freeman (2015) es una de las tecnologías que se impondrá en los próximos años, el número de usuarios aumentará llegando a los mil millones para 2020. Están surgiendo nuevas aplicaciones y dispositivos incluso los que podemos llamar "vestibles" siendo uno de los primeros el IWach.

Actualmente existen numerosas aplicaciones de RA que podemos encontrar en Google Play y otros portales on-line (Prendes, 2015; Cózar, del Moya, Hernández y Hernández, 2015). Aparecen noticias sobre su uso en el ámbito industrial y empresarial, como las pruebas que están realizando Microsoft y Volvo, aplicaciones para amueblar casas, probarnos un vestido o un zapato, libros que muestran imágenes en 3D que podemos girar y que nos llevan a contenidos de video, audio, o texto. Incluso los últimos modelos de Smartphone apuestan por la RA para llegar a todos los consumidores, permitiendo mediante software que se puedan convertir en unas gafas de RA al insertar el móvil en

Tecnologías convergentes para la enseñanza: Realidad Aumentada, BYOD, Flipped Classroom. José Manuel Sánchez-García y Purificación Toledo-Morales. 
una carcasa que lo transforma en un visor de RA mediada por la cámara del dispositivo pudiendo convertirse también en un dispositivo de Realidad Virtual.

Existen varios niveles de RA dependiendo del tipo de dispositivos utilizados y de qué contenidos llevan. Se trata de presentar imágenes o contenidos en $3 \mathrm{~d}$ sobre entornos reales. Para ello de nuevo convergen varias tecnologías, la fotografía, el uso de la red, el modelado y animación de figuras en $3 \mathrm{D}$, la creación de video, y el posicionamiento GPS; son los denominados "niveles de la RA" que fueron definidos por Lens-Fitzgerald (2009) en cuatro niveles (de 0 a 3 ) y que retoma Prendes (2015):

- Nivel 0: Las aplicaciones proporcionan información de mundo físico mediante un hiperenlace, códigos de barras o códigos QR que actúan como lanzadores (triggers). La información aparece en nuestro dispositivo como texto, una página Web, video u otro Formato.

- Nivel 1: Las aplicaciones usan marcadores para el reconocimiento de patrones 2D, dibujos o incluso una fotografía.

- Nivel 2: Se sustituyen el uso de marcadores por el GPS y la brújula de los dispositivos móviles. Superponen el contenido de RA sobre "puntos de interés" sobre lo que vemos a través del dispositivo.

- Nivel 3: Estaría representado por dispositivos como las gafas de RA, la información sobre la realidad esta contextualizada, es inmersiva y personal.

Palmarini, Erkoyuncu, Roy y Torabmostaedi (2018), analizan la aplicación industrial de la RA entre los años 1997-2017 en más de 30 artículos explorando las ventajas e inconvenientes que presentan y concluyendo que en algunas áreas la tecnología de RA aún no está madura, en un ámbito en que la investigación académica comprende más de 50 años. Se pueden encontrar ejemplos en nuestras calles o comercios, siendo su implantación poca y muy lenta, se está empezando a investigar su utilización en los centros educativos de primaria, ESO y Bachillerato, aunque con una mayor presencia en el ámbito universitario debido sus posibilidades de uso. Giasiranis y Sofos (2017) aprecian que mejora la comprensión de los contenidos en el proceso de enseñanza aprendizaje teniendo un efecto positivo en los resultados. Desde la perspectiva de su integración en la escuela Alkhattabi (2017) aborda la implantación de la RA y analiza los beneficios y las dificultades de su implantación así como la aceptación de docentes y alumnos. Otras experiencias como las de Green, Green y Brown (2017) en las aulas de primaria o las realizadas por Pombo, Marques, Carlos, Guerra, Lucas y Loureiro (2018) con grupos de niños de 9-10 años y 13-14 años o la experiencia de Chang y Chung (2018) en la enseñanza de la química son ejemplos del futuro uso de la RA.

Este estudio preliminar busca conocer cómo sería la integración en las aulas de estas tecnologías y metodologías, para lo que es imprescindible el concurso de dispositivos que no pertenecen al centro, si no al alumno. Dentro de lo que se está llamando, Bring Your Own Device (BYOD), Trae tu propio dispositivo, tendencia que se implantará en los próximos años debido a la expansión del uso de dispositivos portátiles (móviles, tablets, laptop) y la mejora y abaratamiento de las redes de telefonía. Las tecnologías que se incorporan al ámbito educativo se pueden utilizar usando el Smartphone o Tablet, en los que ha mejorado la capacidad y para los que existen múltiples aplicaciones. Por ello queremos centrarnos en las tecnologías que están a disposición de

Tecnologías convergentes para la enseñanza: Realidad Aumentada, BYOD, Flipped Classroom. José Manuel Sánchez-García y Purificación Toledo-Morales. 
todos, en manos de los alumnos, de los profesores y que ya se pueden implantar en los centros que disponen de infraestructuras básicas.

\subsection{Uso de BYOD en los centros, una panorámica compleja}

Se considera la implantación de BYOD como una tendencia, que implica el cambio en los medios y en la capacidad de las redes. La Flipped Classroom (Clase Invertida) requiere de acceso a la red y la preparación de contenidos de diversa índole por parte del profesorado, así como la intervención activa del alumno. La RA es un contenido al que accederemos a través de diversos dispositivos, ya sean unas gafas transparentes que sobreponen el contenido aumentado a la imagen real o como un visor, en el que vemos la imagen captada por una cámara. La llegada de esta tecnología aún no se ha producido pese a las expectativas que adelantaba el Informe Horizon (2015) sobre su implantación masiva entre 2018 y 2023, pudiéndose encontrar algunas de las herramientas que se pensaron para ellas en los actuales teléfonos móviles. Existe poca experiencia en la implantación de estas nuevas tendencias en enseñanza, según el Informe Horizon (2015) la integración de BYOD inminente para esta fecha, aun no se ha producido. En recientes investigaciones (Toledo-Morales \& Sánchez-García, 2017) apreciaron que en la mayoría de los centros de ESO y Bachillerato está prohibido el uso de los móviles, el acceso a la red wifi de los centros está limitado o se ha suprimido, y no es factible que los alumnos lleven sus propios dispositivos, ya sean Smartphone o Tablet, a los centros.

\subsection{1. ¿Es positiva la implantación de BYOD?}

Para Johnson, Adams Becker, Estrada y Freeman (2015) el uso de BYOD está relacionado con el aumento de la productividad, más aún cuanto más usuarios trabajan de esta forma. Fomenta una mentalidad de trabajo "on-the-go", en el bus de camino al centro escolar o la universidad, en el tren o en una sala de espera, una biblioteca o cualquier sitio en el que se puede tener acceso a los contenidos usados en el proceso de enseñanza-aprendizaje. Desvincula el aprendizaje del entorno físico o lo sustituye por otro ya sea virtual o de RA. Otro de los aspectos que aumentan la productividad es el de la elección de los dispositivos por los propios usuarios, minimizando la curva de aprendizaje. Es frecuente detectar la presencia de diversos dispositivos de uso cotidiano entre los estudiantes universitarios. Los estudiantes universitarios de nuestro entorno usan sus móviles 3,5 horas al día y tienen más de dos dispositivos (Johnson, Adams Becker, Estrada \& Freeman, 2015). La implantación de BYOD también tendrá repercusiones legales y éticas (Smith, 2017) así como en ámbito de la seguridad y la privacidad de los dispositivos utilizados por el alumnado. Para Song y Wen (2017) el uso de BYOD se ha adoptado en educación, y el uso del propio dispositivo y de las aplicaciones elegidas por el alumno puede contribuir a mejorar el proceso de enseñanzaaprendizaje permitiendo el acceso a la información sin limitaciones de tiempo y lugar. La utilización del propio dispositivo afecta a la conducta y actitud del alumno mostrándose este mas motivado pese a que su uso no está bien regulado en los reglamentos de organización y funcionamiento de los centros educativos (Hopkins, Tate, Sylvester \& Johnstone, 2017).

En la encuesta realizada por el Instituto Nacional de Estadística (2017), sobre Equipamiento y Uso de Tecnologías de Información y Comunicación en los Hogares, queda clara la evolución de la implantación de las TIC, el 83,4 \% de los hogares tiene conexión a internet, y cada vez mas acceden desde fuera de sus casas con dispositivos

Tecnologías convergentes para la enseñanza: Realidad Aumentada, BYOD, Flipped Classroom. José Manuel Sánchez-García y Purificación Toledo-Morales.

Página 5 de 15 
móviles, 91\%. Hay más usuarios de internet que ordenadores y más del $50 \%$ de los españoles accede y tiene presencia en las redes sociales. También nos informa de los aspectos relacionados con la implantación de la tecnología en nuestra sociedad, en los hogares, y como no puede quedar aparte de este fenómeno la escuela. Los hogares con conexión a internet son aproximadamente del 83,4\%, estudios para los que el Instituto Nacional de Estadística (2017) sigue las recomendaciones de EUROSTAT, permite ver la evolución de la implantación pues se realizan anualmente desde al año 2002. Se ha pasado de estudios que hablan de tecnología educativa, en la que los materiales estudiados son retroproyectores, video y cine en el aula, a estudios que se interesan en el uso de los ordenadores, las pizarras digitales o el uso de la red. Estos dispositivos están preparados para instalar APP's que nos dan acceso a los contenidos de RA, lectores de códigos QR o contenidos de RA que podemos generar nosotros mismos. Se puede considerar que la tendencia BYOD facilitará el acceso a entornos de aprendizaje independientes del dispositivo siempre que las infraestructuras que generan los contenidos estén preparadas para este fin, o podemos encontrarnos con alumnos avanzados tecnológicamente que pueden usar dispositivos baratos y amigables para acceder a contenidos fáciles de implementar, y que no pueden úsalos en los ámbitos educativos. Experiencias en la Universidad de Scranton observan que también impactarán en el entorno físico, modificando los objetos muebles de las aulas y la forma de usarlos, si bien esto podrá ocurrir tras un tiempo de implantación y familiarización con los dispositivos (Zahadat, Blessner, Blackburn \& Olson, 2015).

\subsection{Cambios en el rol docente con el Flipped Classroom (FC)}

La introducción de esta tecnología precipitará un necesario cambio en el rol docente para adaptarse a nuevas experiencias en las que media la tecnología. Para autores como Del Moral, Villalustre y Neira-Piñeiro (2016), y Marín y Cabero (2013) la interacción que se produce entre los medios y los alumnos necesita de una guía para saber usarlos en el ámbito educativo y gestionar los contenidos, redefinir el curriculum. González (2006) en trabajos sobre el uso de las TIC, argumenta que los cambios necesarios para esta adaptación necesitan de un esfuerzo en la formación inicial del profesorado, de una formación continua y de una apertura a nuevas ideas e implicación particular cuando se trata de utilizar tecnologías que son novedosas. En esta misma dirección apunta el trabajo de Gisbert (2001) en cuanto a las dificultades de uso de la RV en las aulas con obstáculos similares para la RA, en la preparación de los docentes, el diseño y desarrollo de contenidos, y el uso de entornos tecnológicos. Señala que es necesaria una correcta asunción de nuevos roles entre los que surgen el diseño de contenidos y la evaluación del proceso de enseñanza-aprendizaje con estos medios. Andrus (2014) cree que el BYOD estará presente en los centros educativos en los próximos años, la presencia de dispositivos móviles en la universidad puede ser considerada hoy, y para nuestro entorno actual, como ubicua (y desde hace tiempo). Pese a esta presencia no parece que se encuentre la forma correcta para utilizada en las aulas, los centros universitarios están dotados con redes wifi y muchos de los alumnos ya contratan el acceso a la red en sus dispositivos. El alumnado accede a las aulas con Smartphone, Tablets o portátiles y su uso es potenciado, más que por la universidad, por las compañías de telecomunicaciones, no accediendo en este entorno y con estos dispositivos a contenidos meramente educativos. Otro de los aspectos a tener en cuenta es el de la seguridad y para ello es necesario saber si el acceso a nuestras redes es adecuado. Muchos de estos problemas en la Universidad de Sevilla se resuelven con la

Tecnologías convergentes para la enseñanza: Realidad Aumentada, BYOD, Flipped Classroom. José Manuel Sánchez-García y Purificación Toledo-Morales.

Página 6 de 15 
conexión a Eduroam, red wifi controlada por la propia universidad y que se puede considerar suficientemente segura. Si se considera que la implantación de BYOD es una tendencia, no un dispositivo concreto, el uso de estos elementos se normaliza. El acceso a una red wifi, que hace diez años era considerado como algo superfluo actualmente es un servicio más, puede que incluso más necesario que otros (Helman, 2014). Se considera que esta confluencia se viene produciendo ya entre los dispositivo móviles y las redes, al principio con redes menos potentes y portátiles o PDA, hoy con móviles y Tablets, mañana con gafas de RA y contenidos 3D. Se están implantando en los entornos profesionales y de ocio, y han de integrarse en el ámbito educativo. No se ha comenzado a entender, ni se ha implantado aun el uso de los BYOD y Herman (2014) ya empiezan a hablar procesos que pasan por que las conexiones no sean proporcionadas por los centros sino contratadas por los alumnos.

Cabe preguntarse si el uso de medios nos lleva hacia la implantación de FC, cambio notable en cuanto al rol del profesor en las aulas y una forma novedosa de impartir la docencia. Según el Informe Horizon (2015) esta tendencia se irá implantando en los niveles superiores de enseñanza dotando a los estudiantes y profesores de herramientas adecuadas no presenciales mediante las que presentar los contenidos y los conocimientos, dejando el tiempo de clase para la resolución de las dudas que surjan y para profundizar en la materia de la que ya tienen un conocimiento previo, ya sea por presentaciones, lecturas recomendadas o fragmentos de video realizados ad hoc. Existen voces que no están completamente de acuerdo con esta implantación y que consideran como Flumerfelt y Green (2013) que apoyarse en el uso de FC es un riesgo para los estudiantes. Pese a ello, el uso de FC en las aulas está siendo abordado desde diferentes ámbitos de estudio, como la experiencia en educación física abordado por Gómez, Castro y Toledo-Morales (2015). Fulton (2012) se enfoca en cuestiones de seguridad y en los riesgos que conlleva esta implantación y que condicionan la aceptación por parte de las instituciones educativas, si bien la reducción de costos y la mejora del rendimiento atraen e interesan a los centros.

El FC se está implantando en todos los ámbitos educativos, desde las enseñanzas de Matemática hasta las de Bellas Artes, percibiéndose ventajas en cuanto a participación activa y rendimiento académico de los alumnos. Para Lo, Hew y Chen (2017), en el FC inciden el tipo de actividad realizada fuera del aula, su efecto en el aprendizaje del alumno, la percepción de los participantes y el reto de utilizarla con las complejidades que conlleva. Encontrando estos autores beneficioso el uso del tiempo en clase que permite realizar las actividades, corregirlas y mejorarlas al estar supervisadas por el docente. En la enseñanza de Bellas Artes Shu, Li y Cao (2017), analizan su uso para innovar y mejorar utilizando FC y entornos de red, percibiendo que un mejor desarrollo del alumno y su adecuación a este tipo de enseñanzas. En el FC también puede integrar el uso BYOD, Hung (2017) "gamifica" el aula usando el programa Clikers y los propios dispositivos BYOD. Experiencia que tuvo resultados muy positivos en cuanto a la mejora académica y percepción de los alumnos. 


\section{Seguridad y accesibilidad, retos para una implantación tecnológica en las aulas}

En este proceso de implantación nos encontramos con problemas e inconvenientes que el mundo de la empresa ya ha abordado y que empiezan a estar presentes en el ámbito educativo. El más significativo es el del control sobre el uso de los dispositivos y de si las tareas para las que se usan en el aula están relacionadas con las materias, trabajos o investigaciones que vamos a realizar, pudiendo producirse derivas que podemos considerar desaconsejables. Desde el uso de los dispositivos para visionar paginas inadecuadas, recibir el correo particular o mantener una conversación on-line. Muchos de estos usos surgen al utilizar el alumno un dispositivo de su propiedad, por lo que se deberían ponderar cómo se introduce su uso en el aula y así mejorar los procesos de enseñanza-aprendizaje.

Para Disterer y Kleiner (2013) los dispositivos que son propiedad del alumnado favorecen el uso, aumentado la comodidad y minimizando la curva de aprendizaje en su manejo. Pero también aumentando la aparición de problemas técnicos, que los gestores de redes y especialistas en seguridad informática han denominado como "Anarquía informática", y que ha llevado a que reutilicen las siglas BYOD como "Bring your own danger". Aspectos que llevan a la necesidad de reglamentar y encapsular (protegiéndolos de alteraciones e intrusiones no deseadas) los entornos de trabajo, ya sean en las empresas o en la universidad y los distintos entornos educativos, más necesario aun cuando se trata de clases como primaria, secundaria y bachillerato en los que se tendrá que ser extremadamente cuidadoso.

El uso de dispositivos de los propios alumnos se presenta como ventajoso, se pueden usar en cualquier lugar y en cualquier momento, pero esta misma ventaja elimina los límites entre un uso privado y un uso académico, flexibilizando horarios y potenciado la capacidad de realizar trabajos o recabar información sobre un tema en cualquier lugar. Este mismo uso amplio en entornos que podemos considerar no seguros, hace que sea necesario mejorar en estos aspectos, que ya se han considerado en el mundo empresarial por diversos motivos y que podemos adecuar al ámbito educativo aprendiendo de experiencias previas. Por esta misma y otras razones de protección de datos de profesores y alumnos, teniendo en cuenta la complejidad presente en nuestras instituciones y que "la complejidad es el enemigo de la seguridad", es necesario respetar especialmente la confidencialidad, integridad y autenticidad (Disterer \& Kleiner, 2013). Como solución se presentan varias opciones: el uso de una partición aislada en el dispositivo; el uso de aplicaciones visualizadas en determinados dispositivos y que se ejecutan en servidores propios, requiere más ancho de banda y más inversión; o trabajar los contenidos en la nube que requiere de la conexión, física o mediante wifi, de los dispositivos.

Diversos autores como Flumerfelt y Green (2013) aprecian que las empresas han potenciado el uso de BYOD y que en palabras de Venkatesh y Davis, (2000) su adopción representa un riesgo menor que el de no usarlos, haciendo que la productividad no mejore, en lo que estos autores determinan como un equilibrio entre los beneficios y los riesgos que comporta esta implantación. Si bien en el ámbito empresarial prima el beneficio, en los ámbitos educativos se sobredimensionan los riesgos. Se han de evaluar las expectativas, la disminución de la curva de aprendizaje en

Tecnologías convergentes para la enseñanza: Realidad Aumentada, BYOD, Flipped Classroom. José Manuel Sánchez-García y Purificación Toledo-Morales.

Página 8 de 15 
el uso de software, la utilidad de los programas educativos, el abaratamiento de costes y la sencillez de manejo de los dispositivos; y los riesgos que presenta el uso de un sistema informático complejo, la aparición de virus y malware, la perdida de datos, el acoso en las redes sociales, el miedo de los docentes a aceptar el cambio o la pérdida de autoridad del profesor frente a las redes (De las Cuevas, Mora, Merelo, Castillo, GarcíaSánchez \& Fernández-Ares, 2015; D'Arcy, 2011; Niehaves, Ortbach \& Koeffer, 2012; Flumerfelt \& Green, 2013; Leclercq-Vandelannoitte, 2015; Vignesh \& Asha, 2015). Es necesario buscar formas de implantación y uso que en el ámbito educativo han de primar la formación de los alumnos, el uso de las redes para obtener información, el uso de esta información y su influencia en los diferentes aspectos del proceso de enseñanza aprendizaje. Sawyer y Winter (2011) argumentan que las investigaciones sobre el uso de tecnologías informáticas se han centrado más en la empresa y en la seguridad desde el punto de vista de los profesionales centrados en la productividad, rentabilidad y seguridad, dejando fuera la incorporación de prácticas innovadoras y los usos alternativos de estas nuevas tendencias. Siendo estos temas importantes para la educación no son, ni deben ser, el foco principal de los estudios e investigaciones realizados en nuestro ámbito.

En cuanto a las formas del uso de las tecnologías y las medidas que se pueden adoptar para su implantación y las diversas estrategias que se presentan Harris, Ives y Junglas (2011) analizan tres comportamientos que son comunes en diferentes ámbitos. Un comportamiento anárquico, en el que se permite la entrada de dispositivos sin ningún tipo de restricciones; autoritario, que restringe el número y tipo de dispositivos que pueden acceder y de adopción, que asume su uso y lo considera beneficioso. LeclercqVandelannoitte (2015) ven como beneficios la rentabilidad tecnológica de un uso normal y cotidiano de la tecnología ya que presenta un acceso continuo a la información. Muchos expertos en seguridad piensan que esta situación se da por que las personas encargadas del acceso de los dispositivos, contenidos y datos no perciben riesgos ni amenazas sobre sus sistemas. Los que si aprecian estas complicaciones y creen que han de ser controlados los accesos a los contenidos y los dispositivos desde las que se realizan así como buscar una gestión controlada y regulada que tenga en cuenta el potencial de uso de estas tendencias en conjunto. Estos mismos autores plantean tres posturas que pueden tomar las instituciones. Primera, de inducción, las institución actúa de forma proactiva para implantación y uso de tecnologías como BYOD, RA o FC; esta debería de ser la postura de las universidades como fuente de potenciación del conocimiento y pionera en el uso de las tecnologías convergentes en el espacio educativo. Segunda, de normalización, en esta situación la institución aprecia en la incorporación y uso estos medios un proceso normal, parte de las practicas diarias. Y por último, un proceso de regulación en la que se aprecian tanto oportunidades como riesgos, aspecto que lleva a la toma de decisiones para la implantación de normas de uso y de dispositivos autorizados para el mismo (Leclercq-Vandelannoitte, 2015).

\section{Conclusiones}

La implantación de estos dispositivos es casi inevitable en nuestro entorno. Su aparición para el uso y visualización de la RA y RV es otro paso que parece se impone en nuestras sociedades. Parece obvio que la implantación no está procediendo de la voluntad

Tecnologías convergentes para la enseñanza: Realidad Aumentada, BYOD, Flipped Classroom. José Manuel Sánchez-García y Purificación Toledo-Morales.

Página 9 de 15 
legislativa o política, al menos en educación, y que está ligada a las empresas y a la generalización del uso de dispositivos móviles, a la iniciativa de particulares, profesores e investigadores. APP's como la de una conocida marca de pintura nos permiten ver el color de nuestras paredes antes de pintarlas, se instalan en un minuto, y en los treinta segundos siguientes ya estamos utilizándolas. El catálogo de una marca mundial de muebles permitirá insertar 90 de sus artículos en nuestro hogar mediante el uso de RA, ver cómo queda una nueva cocina o estar dentro de una RA de $180^{\circ}$ o $360^{\circ}$ para explorar una habitación, pueden darnos indicaciones de cómo crear contenidos de RA amigables adecuados para su uso intuitivo por los alumnos.

Ejemplo de esta confluencia lo podemos encontrar en la experiencia realizada por la Universidad de Sevilla en la página http://intra.sav.us.es/proyectorafodiun/, en la que colaboran numerosos profesores de varias universidades: País Vasco, Jaén, Alicante, Santiago de Compostela, Pablo de Olavide, Córdoba, Autónoma de Madrid, Málaga y Sevilla; que introducen al alumno en el uso de la RA en el entorno educativo. Existe obstáculos para su integración en la escuela como la escasa preparación para su uso por parte del profesorado o la imposibilidad de que los alumnos aporten sus propios dispositivos por estar prohibidos en los centros. No existe acceso a contenidos de forma fácil, y la posibilidad de crearlos es compleja y se presenta aún lejana. Aunque están apareciendo en el mercado nuevos programas relativamente fáciles de utilizar y de gran potencia para crearlos. El gasto para la adquisición de dispositivos, que en principio puede parecer caro, se abaratará en los próximos años.

El primer uso que se hace de estos elementos no es educativo, proviene del ámbito empresarial, tiendas que ofrezcan sus productos mediante RA. Enfocado a un uso lúdico con distintos desarrollos por parte de compañías de videojuegos que integran el uso de RV y RA, por lo que el costo de la integración en los hogares pasa por las consolas de videojuegos y estos costes serán asumidos por las familias con fines lúdicos. Porque no para un uso educativo o para su implantación y uso en la escuela. Por otra parte existen riesgos de seguridad para los alumnos de primaria y de secundaria, acceso a contenidos inapropiados, a juegos de ordenador en red o acoso en redes sociales. Aspectos que tendrían que regularse y que requerirían de una especial vigilancia por parte del profesorado en estas etapas y de acceso a entornos seguros en los centros que no les permitieran salirse de las paginas o contenidos educativos desarrollados para este fin, para lo que sería necesario un servidor en el propio centro o el acceso a contenidos seguros gestionados por la administración.

En estadios iniciales no sería necesaria la implantación de BYOD para tener acceso a contenidos de RA. El uso del ordenador del profesor permitiría el acceso a contenidos de RA que se podrían visualizar en la PDI o a través de un proyectos de video dejando el uso de BYOD para etapas superiores de educación en la que los alumnos estarían más maduros. El aspecto novedoso de plantear la convergencia entre BYOD y RA puede no ser bien comprendido por los docentes, que pueden llegar a pensar que es más un artificio que una posibilidad de usar una tecnología muy avanzada en el aula. Otro aspecto importante es la posibilidad de que los alumnos superen al profesorado en cuanto a las competencias necesarias para su uso, por lo que se pueden sentir poco dispuestos a que se produzca esta integración. Observamos que con la tecnología actual sería interesante y posible la implantación de contenidos de RA en las aulas. No 
requeriría de una gran inversión, solo la adecuación de la infraestructura existente. Si bien el uso y la implantación de gafas de RA serían deseables, habría que esperar su abaratamiento y producción en masa, como se ve en expectativas para el 2020-23. Momento en el que se esperan que sustituyan a los Smartphone, aspecto que veremos si llega a cumplirse o no, o se conjugaran ambos elementos. La sustitución de los elementos del aula y la integración de materiales curriculares basados en RA es una oportunidad. Los autores de este trabajo aprecian que la convergencia entre BYOD, RA y FC es deseable y que aportaría contenidos y formas de impartir docencia novedosa, y mejoras en todos los ámbitos y niveles educativos. Su convergencia es posible, con los medios actuales y con los medios que se presentan como interesantes para los próximos años aún más, pudiendo llegar a suponer un cambio radical en el uso de la tecnología en las aulas.

Presentación del artículo: 24 de julio de 2017

Fecha de aprobación: 7 de diciembre de 2017 Fecha de publicación: 22 de diciembre de 2017

Sánchez-García, J.M., y Toledo-Morales, P. (2017). Tecnologías convergentes para la enseñanza: Realidad Aumentada, BYOD, Flipped Classroom. RED. Revista de Educación a Distancia, 55. Consultado el (dd/mm/aaaa) en http://www.um.es/ead/red/55/sanchez_toledo.pdf

\section{Financiación}

Esta investigación no ha recibido ninguna subvención específica de los organismos de financiación en los sectores públicos, comerciales o sin fines de lucro.

\section{Referencias bibliográficas}

Alkhattabi (2017) Augmented Reality as E-learning Tool in Primary Schools' Education: Barriers to Teachers' Adoption. International Journal of Emerging Technologies in Learning, 12, (2), 91-100. https://doi.org/10.3991/ijet.v12i02.6158

Andrus, F. (2014). Preparing for the BYOD invasion on your campus. University Business Magazine. Recuperado de http://www.universitybusiness.com/article/preparing-byod-invasion-yourcampus

Azuma, R. (1997). A Survey of Augmented Reality. Presence: Teleoperators and Virtual Environments, 6 (4), 355-385. http://dx.doi.org/10.1162/pres.1997.6.4.355 
Barfield, W. (2015). Wearable Computers and Augmented Reality. Musings and Future Directions. En W. Barfield (Ed.), Fundamentals of Wearable Computers and Augmented Reality, (pp.3-12). CRC Press, Taylor \&Francis Group.

Basogain, X., Olabe, M., Espinosa, K., Rouèche, C., \& Olabe, J.C. (2007). Realidad Aumentada en la Educación: Una tecnología emergente. Actas Online Educa Madrid 2007. $7^{a}$ Conferencia Internacional de la Educación y Formación basada en las Tecnologías, (pp. 24-29), Madrid.

Chang, R.-C., Chung, L.-Y. (2018). Integrating augmented reality technology into subject teaching: The implementation of an elementary science curriculum. Lecture Notes in Electrical Engineering, 422, 187-195.

Cochrane, T., Antonczak, L., Keegan, H., \& Narayan, V. (2014). Riding the wave of BYOD: developing a framework for creative pedagogies. Research in Learning Technology, 22, 1-14. http://dx.doi.org/10.3402/rlt.v22.24637

Cózar Gutiérrez, R., del Moya Martínez, M., Hernández Bravo, J.A., Hernández Bravo, J.R. (2015). Tecnologías emergentes para la enseñanza de las ciencias sociales. Una experiencia con el uso de realidad aumentada en la formación inicial de maestros. Digital Education Review, 27, 138-153. Recuperado de http://revistes.ub.edu/index.php/der/article/view/11622/pdf

D'Arcy, P. (2011). CIO Strategies for Consumerization: The Future of Enterprise Mobile Computing. Dell CIO Insight Series. Recuperado de http://marketing.dell.com/Global/FileLib/hp_microsite/dell-consumerization.pdf

De las Cuevas, P., Mora, A.M., Merelo, J.J., Castillo, P.A., García-Sánchez P., \& Fernández-Ares, A. (2015). Corporate security solutions for BYOD: A novel user-centric and self-adaptive system. Computer Communications, 68, 83-95. http://dx.doi.org/10.1016/j.comcom.2015.07.019

De Pedro, J. (2011). Realidad Aumentada: un nuevo paradigma en la educación superior. En E. Campo, M. García, E. Meziat \& L. Bengochea (eds.), Educación y Sociedad (pp. 300-307). Chile: Universidad La Serena.

Del Moral, M.E., Villalustre, L. \& Neira-Piñeiro, M.R. (2016). Minors trapped in the magical world of augmented reality, advergaming and social networks. Prisma Social, número Especial 1, 1-28. Recuperado de http://revistaprismasocial.es/article/view/1312/1376

Durlach, N.I., \& Mavor, A. S. (1995).Virtual Reality: Scientific and Technological Challenges. Washington, D.C: National Academy Press.

Flumerfelt, S., \& Green, G. (2013). Using Lean in the Flipped Classroom for at Risk Students. Educational Technology and Society, 16 (1), 356-366. Recuperado de http://www.ifets.info/journals/16_1/31.pdf

Tecnologías convergentes para la enseñanza: Realidad Aumentada, BYOD, Flipped Classroom. José Manuel Sánchez-García y Purificación Toledo-Morales. 
Fulton, K.P. (2012). 10 Reasons to Flip. Phi Delta Kappan, 94 (2), 20-24. http://dx.doi.org/10.1177/003172171209400205

Giasiranis, S., \& Sofos, L. (2017). Flow Experience and Educational Effectiveness of Teaching Informatics using AR. Educational Technology \& Society, 20 (4), 7888. Recuperado de http://www.jstor.org/stable/26229207

Gisbert, M. (2001). Nuevos roles para el profesorado en entornos digitales. En J. Salinas \& A. Batista (Coord.), Didáctica y Tecnología Educativa para una Universidad en un Mundo Digital (pp. 65-85). Panamá: Universidad de Panamá. Facultad de Ciencias de la Educación.

Gómez, I., Castro, N., \& Toledo-Morales, P. (2015). Las Flipped Classroom a través del Smartphone: Efectos de su experimentación en Educación Física Secundaria. Prisma Social, 15, 296-351. Recuperado de http://www.isdfundacion.org/publicaciones/revista/numeros/15/secciones/temati ca/pdf/t_09_flipped-classroom_296-351.pdf

González, J.C. (2006). B-learning utilizando software libre, una alternativa viable en educación superior. Revista Complutense de Educación, 17 (1), 121-133.

Green, J., Green, T., \& Brown, A. (2017). Augmented Reality in the K-12 Classroom. TechTrends, 61 (6), 603-605. Recuperado de https://www.springerprofessional.de/en/augmented-reality-in-the-k-12classroom/15034986

Harris, J.G., Ives, B., \& Junglas, I. (2011). The Genie is Out of the Bottle: Managing the Infiltration of Consumer IT into the Workforce. Accenture Institute for High Performance. http://nstore.accenture.com/IM/FinancialServices/AccentureLibrary/data/pdf/gen ie-out-of-bottle-it-workforce.pdf

Helman, B. (2014). Bring Everything: BYOD's Evolution In Higher Education. Recuperado de http://www.informationweek.com/interop/bring-everythingbyods-evolution-in-higher-education/d/d-id/1114042

Hopkins, N., Tate, M., Sylvester, A., \& Johnstone, D. (2017). Motivations for 21st century school children to bring their own device to school. Information Systems Frontiers, 19 (5), 1191-1203. https://doi.org/10.1007/s10796-016-9644-z

Hung, H.-T. (2017). Clickers in the flipped classroom: bring your own device (BYOD) to promote student learning. Interactive Learning Environments, 25(8), 983-995. https://doi.org/10.1007/s10796-016-9644-z

Instituto Nacional de Estadística (2015). Encuesta sobre equipamiento y uso de tecnologías de información y comunicación en los hogares. Madrid: INE. Recuperado 
http://www.ine.es/dyngs/INEbase/es/operacion.htm?c=Estadistica_C\&cid=1254 $736176741 \&$ menu=ultiDatos\&idp $=1254735976608$

Johnson, L., Adams Becker, S., Estrada, V., \& Freeman, A. (2015). NMC Horizon Report: Edición Educación Superior 2015. Austin, Texas: The New Media Consortium.

Leclercq-Vandelannoitte, A. (2015). Managing BYOD: how do organizations incorporate user- driven IT innovations?. Information Technology \& People, 28(1), 2 - 33. http://dx.doi.org/10.1108/ITP-11-2012-0129

Lens-Fitzgerald, M. (2009). Augmented Reality Hype Cycle. Recuperado de http:// www.sprxmobile.com/the-augmented-reality-hype-cycle

Lo, C.K., Hew, K.F., \& Chen, G. (2017). Toward a set of design principles for mathematics flipped classrooms: A synthesis of research in mathematics education. Educational Research Review, 22, 50-73. https://doi.org/10.1016/j.edurev.2017.08.002

Marín, V., \& Cabero. J. (2013). Introducción a la Educación mediática. Córdoba, Universidad de Córdoba. En V. Marín (Coord.), Educación mediática y dimensión educativa de las TIC (pp. 3-22). Córdoba: Servicio de Publicaciones de la Universidad de Córdoba.

Niehaves, B., Koeffer, S. \& Ortbach, K. (2012). IT consumerization - a theory and practice review. AMCIS 2012 Proceedings. Paper 18. Seattle, WA. Recuperado de http://aisel.aisnet.org/amcis2012/proceedings/EndUserIS/18

Palmarini, R., Erkoyuncu, J.A., Roy, R., \& Torabmostaedi, H. (2018). A systematic review of augmented reality applications in maintenance. Robotics and Computer-Integrated Manufacturing, 215-228, https://doi.org/10.1016/j.rcim.2017.06.002

Peña, S., Lazkano, I., \& García, D. (2016). La transición digital de los diarios europeos: nuevos productos y nuevas audiencias. Comunicar, XXIV (46), 27-36. http://dx.doi.org/10.3916/C46-2016-03

Pombo L., Marques M.M., Carlos V., Guerra C., Lucas M., \& Loureiro M.J. (2018). Augmented Reality and Mobile Learning in a Smart Urban Park: Pupils' Perceptions of the EduPARK Game. In Ó. Mealha, M. Divitini, \& M. Rehm (Eds.), Citizen, Territory and Technologies: Smart Learning Contexts and Practices. SLERD 2017. Smart Innovation, Systems and Technologies, 80, (pp. 90-100). Springer, Cham. http://dx.doi.org/10.1007/978-3-319-61322-2_9

Prendes, C. (2015). Realidad Aumentada y Educación: Análisis de experiencias prácticas. Pixel-Bit, 46, 187-203. doi: http://dx.doi.org/10.12795/pixelbit.2015.i46.12 
Sawyer, S., \& Winter, S.J. (2011). Special issue on futures for research on information systems: prometheus unbound?. Journal of Information Technology, 26 (2), 9598. http://dx.doi.org/10.1057/jit.2011.7

Smith, W.P. (2017). Can we borrow your phone? Employee privacy in the BYOD era. Journal of Information, Communication and Ethics in Society, 15 (4), 397-411. https://doi.org/10.1108/JICES-09-2015-0027

Song, Y., \& Wen, Y. (2017). Integrating Various Apps on BYOD (Bring Your Own Device) into Seamless Inquiry-Based Learning to Enhance Primary Students' Science. Learning Journal of Science Education and Technology, 26 (126), 112. https://doi.org/10.1007/s10956-017-9715-z

Shu, L., Li, G., \& Cao, X. (2017). Research on the optimization and innovation of art flipped classroom teaching based on the network environment. Technical Bulletin, 55 (11), 672-678. Recuperado de http://www.boletintecnico.com/index.php/bt/article/view/1114/1118

Toledo-Morales, P. \& Sánchez-García, J. (2017). Realidad Aumentada en Educación Primaria: efectos sobre el aprendizaje. Revista Latinoamericana de Tecnología Educativa, 16 (1), 79-92. Recuperado de http://relatec.unex.es/article/view/2810/2004

Venkatesh, V., \& Davis, F.D. (2000). A theoretical extension of the technology acceptance model: four longitudinal field studies. Management Science, 46 (2), 186-204. Recuperado de http://pubsonline.informs.org/doi/abs/10.1287/mnsc.46.2.186.11926?journalCod $\mathrm{e}=\mathrm{mnsc}$

Vignesh, U, \& Asha, S. (2015). Modifying security policies towards BYOD. 2nd International Symposium on Big Data and Cloud Computing (ISBCC'15). Procedia Computer Science, 50, 511-516. http://dx.doi.org/10.1016/j.procs.2015.04.023

Zahadat, N., Blessner, P., Blackburn, T., \& Olson, B.A. (2015). BYOD security engineering: A framework and its analysis. Computers \& Security, 55, 81-99. http://dx.doi.org/10.1016/j.cose.2015.06.011 